\title{
Transthoracic echocardiography in the assessment of cardiogenic causes of ischaemic stroke
}

\author{
Wojciech Król' ${ }^{1}$, Anna Żarek², Aleksandra Wilk², Wioletta Guzik², Marlena Ziemska², \\ Marcin Konopka ${ }^{1}$, Magdalena Franczuk-Gwiazda ${ }^{3}$, Marek Kuch ${ }^{4}$, Wojciech Braksator ${ }^{1}$ \\ ${ }^{1}$ Medical University of Warsaw, 2nd Medical Faculty, Department of Sports Cardiology \\ and Noninvasive Cardiovascular Imaging, Warsaw, Poland \\ ${ }^{2}$ Medical University of Warsaw, Warsaw, Poland \\ ${ }^{3}$ Masovian, Specialist Health Centre, 9th Department (Neurology), Pruszkow, Poland \\ ${ }^{4}$ Medical University of Warsaw, Department of Cardiology, Hypertension and Internal Diseases, Warsaw, Poland
}

\begin{abstract}
Introduction. One of the leading causes of death in Poland is stroke. Cardiogenic stroke is known to be one of the most important reasons for acute ischaemic stroke (AIS), comprising $25-30 \%$ of all AISs.

Aim of study. Assessment of the prevalence of different risk factors of cardiogenic causes of AIS using transthoracic echocardiography (TTE)

Material and methods. Transthoracic echocardiograms performed in patients with AIS admitted to a single neurological ward between October 2013 and September 2017 were analysed. Patients were assigned, based on the results of their TTE and their previous medical history of atrial fibrillation (AF), to one of three groups depending on the level of the risk of occurrence of cardiogenic causes of AIS.

Ethical permission. According to Dz.U.2001, no. 126, 1381 no ethical permission was needed.

Results. 663 patients with AIS were included in the study. Patients with high risk of cardiogenic cause of AIS: $26.7 \%$ ( $=177$ patients [p]). Of these, $64.4 \%$ (114 p) were diagnosed with AF. 31.6\% (56 p) with sinus rhythm during hospitalisation had a history of paroxysmal AF (PAF). In $11.9 \%$ (21 p) of the patients qualified to the high risk group, factors other than AF were found. Patients with moderate risk of cardiogenic cause of AIS: $10.1 \%$ (67 p). Patients with low risk of cardiogenic cause of AIS: $25.9 \%$ (172 p). Echocardiographic results led to a change in therapy in $1.21 \%$ of cases.

Conclusions. 1. Transthoracic echocardiography performed routinely in all AIS patients affects the treatment in a very low percentage of cases. 2. The group that could benefit the most from TTE examination includes people without established indications for chronic anticoagulant therapy, in particular patients after myocardial infarction and people with additional clinical symptoms. 3. In patients with AIS, the diagnostic sensitivity of TTE in the detection of PFO is low. Young people with a cryptogenic ischaemic stroke should undergo a transoesophageal assessment.
\end{abstract}

Key words: stroke, atrial fibrillation, transthoracic echocardiography, cardiogenic stroke, echocardiography (Neurol Neurochir Pol 2019; 53 (2): 156-161)

\section{Introduction}

Strokes are one of the leading causes of death worldwide [1]. It is estimated that ischaemic strokes (AIS) account for $87 \%$ of all strokes [2]. One of the most common causes of strokes is cardiogenic stroke (25-30\% of all AIS) [3]. By definition, a cardiogenic stroke is caused by embolic material entering the cerebral circulation from the heart cavities. This material

Address for correspondence: Wojciech Król, Medical University of Warsaw, 2nd Medical Faculty, Department of Sports Cardiology and Noninvasive Cardiovascular Imaging, Warsaw, Poland, e-mail: wukrol@gmail.com 
may be thrombi formed as a result of blood flow through the heart, or foreign bodies present in the heart, as well as fragments of tumours or bacterial vegetation. Pathologies that are most often associated with the formation of embolic material in the heart include atrial fibrillation and other conditions associated with the release of blood flow disturbing Virchow's triad with an increased risk of thrombus formation such as aneurysms and post-infarction scars, degenerative valve changes, in particular in the course of rheumatic heart disease, and a non-compacted myocardium.

The risk of a cardiogenic stroke can be divided into several groups, depending on the cause. $[3,4]$. The basic examination by which patients can be qualified for a particular risk group is transthoracic echocardiography (TTE). This is recommended for every patient with a suspected cardiogenic stroke [5]. Potentially the greatest benefit of TTE can be experienced by patients in whom the detection of the source of a cardiogenic stroke during the examination would involve a change in the treatment procedure. This group includes patients with thrombi in the left heart cavities and patients with previously undiagnosed atrial fibrillation, in whom the introduction of an anticoagulant therapy significantly reduces the risk of a subsequent stroke [6]. Due to the high mortality rate, which is $10-17 \%$ within 14 days [7], and a high relapse rate, it is important to detect the cause of a cardiogenic stroke early and to implement the correct treatment.

\section{Clinical rationale for the study}

Assessment of the occurrence of cardiogenic risk factors for the source of acute ischaemic stroke using transthoracic echocardiography.

Assessment of the effect of transthoracic echocardiographic results on treatment change.

\section{Materials and methods}

This retrospective analysis included the results of echocardiographic examinations carried out in consecutive patients hospitalised at the Neurological Ward and Stroke Ward with suspected AIS between October 2013 and September 2017. Echocardiography was performed in all patients with initially suspected AIS. A total of 717 studies were analysed. The tests were carried out using Philips Ultrasound Inc. HD11 XE (USA). Echocardiographic measurements and ranges of norms were applied in accordance with generally accepted standards [4]. The physician who performed the echocardiographic examination subjectively assessed study conditions to be 'normal', 'difficult' or 'very difficult' according to the available acoustic window and patient cooperation leading to adequate visualisation of all cardiac structures. Technical difficulties during the examination resulted mainly from anatomical conditions, lack of cooperation, and difficulties in understanding the process in some patients, plus related problems such as the patient's optimal position for the examination. Due to patients' immobilisation and the related difficulty in assessing biometric parameters (height and weight) that would allow the physician to calculate body surface, non-indexed left-ventricular mass (LVM) was used to assess left ventricular hypertrophy (standard: up to $150 \mathrm{~g}$ for women, up to $200 \mathrm{~g}$ for men), and for the size of the left atrium - the surface area in a four-chamber projection (standard: $18.9 \mathrm{~cm}^{2}$ for women; $20.3 \mathrm{~cm}^{2}$ for men) [8]. A concentric hypertrophy was considered when the LVM was above normal at the relative thickness of the left ventricle $(\mathrm{RWT})>0.42$. The possibility of diagnosing hypertrophic cardiomyopathy was determined in accordance with current recommendations in patients with a thickness of the interventricular septum $>1.5 \mathrm{~cm}$ [9]. Dilated cardiomyopathy was defined as a significant enlargement of the left ventricular end-diastolic diameter (LVEDd) of $>6.0 \mathrm{~cm}$ coexisting with left ventricular ejection fraction $(\mathrm{EF})<40 \%$ [10]. All echocardiographic examinations were made by one experienced echocardiographer.

The occurrence of AF was assessed based on medical history, ECG examination descriptions, and Holter's ECG tests performed during the hospitalisation.

Based on TTE results and data obtained from medical records, patients were assigned to one of three risk groups of the cardiogenic stroke source:

- High risk group - HR

- Moderate risk group - MR

- Low risk group - LR

The qualification criteria were developed based on literature data. Neurologic and cardiologic (echocardiographic) recommendations are inconsistent $[11,12]$. Some divide the risk of cardiogenic sources of embolic stroke into two groups (high and moderate) [4], while others divide it into three (high, moderate, and low [3]). Due to the lack of consensus on this subject, qualification criteria for the above-mentioned divisions were combined and are presented in Table 1 [11, 13-16] Statistica version 13 (Statsoft) was used for statistical analysis. The results are presented as mean $( \pm)$ standard deviations, and the frequency of occurrence of qualitative variables is given as a percentage. The analysis of variance (ANOVA) using post hoc Shaffe and NIR tests was used to assess the significance of differences between the three analysed subgroups of patients with current risk factors for cardiogenic stroke. The level of statistical significance was considered $\mathrm{P}<0.05$.

\section{Results}

The initial analysis included 717 descriptions of TTE research. 54 research descriptions were excluded from the research for the following reasons:

- after the analysis of discharge cards, due to the lack of a definitive diagnosis of ischaemic stroke (49 people),

- due to the patient's death before a definitive diagnosis of ischaemic stroke (three people), 
Table 1. Risk of cardiogenic embolism depending on underlying cause

\begin{tabular}{|c|c|c|}
\hline HIGH (HR) & MODERATE (MR) & LOW (LR) \\
\hline atrial fibrillation & spontaneous echo contrast in heart chambers & patent foramen ovale \\
\hline mechanical artificial heart valves & $\begin{array}{l}\text { severe or moderate degenerative changes in } \\
\text { left heart valves }\end{array}$ & atrial septal aneurysm \\
\hline rheumatic heart disease & biological artificial heart valve & prolapse of the mitral valve \\
\hline thrombus in the heart chambers & post-infarction left ventricular aneurysm & features of post-infarction scar (without aneurysm) \\
\hline infective endocarditis & dilated cardiomyopathy & left ventricular hypokinesia \\
\hline $\begin{array}{l}\text { non-compaction of the myocardium } \\
\text { myxoma }\end{array}$ & mitral annular calcification (MAC) & hypertrophic cardiomyopathy \\
\hline
\end{tabular}

Table 2. Echocardiographic parameters

\begin{tabular}{|c|c|c|c|c|}
\hline & $\begin{array}{l}\text { Low risk of cardiogenic } \\
\text { stroke }\end{array}$ & $\begin{array}{l}\text { Medium risk of cardio- } \\
\text { genic stroke }\end{array}$ & $\begin{array}{l}\text { High risk of cardiogenic } \\
\text { stroke }\end{array}$ & $P$ (ANOVA) \\
\hline Age (years) $\pm S D$ & $70.5( \pm 13)$ & $78.6( \pm 10)$ & $77.9( \pm 11)$ & $<0.001 ¥^{*}$ \\
\hline Women (\%) & 37.8 & 50.7 & 57.1 & $<0.001 ¥$ \\
\hline $\operatorname{IVSd}(\mathrm{cm}) \pm \mathrm{SD}$ & $1.36( \pm 0.3)$ & $1.34( \pm 0.2)$ & $1.30( \pm 0.2)$ & NS \\
\hline $\operatorname{LVED}(\mathrm{cm}) \pm \mathrm{SD}$ & $4.83( \pm 0.7)$ & $4.80( \pm 0.8)$ & $4.81( \pm 0.8)$ & NS \\
\hline $\mathrm{LV}$ mass $(\mathrm{g}) \pm \mathrm{SD}$ & $258( \pm 89)$ & $252( \pm 102)$ & $244( \pm 88)$ & NS \\
\hline $\mathrm{RWT} \pm \mathrm{SD}$ & $0.53( \pm 0.1)$ & $0.53( \pm 0.1)$ & $0.52( \pm 0.1)$ & NS \\
\hline$E F(\%) \pm S D$ & $56.3( \pm 8.3)$ & $53.1( \pm 12)$ & $54.4( \pm 9.9)$ & $P=0.02^{*}$ \\
\hline $\operatorname{LAd}(\mathrm{cm}) \pm \mathrm{SD}$ & $4.16( \pm 0.6)$ & $4.21( \pm 0.6)$ & $4.56( \pm 0.7)$ & $P<0.001 ¥ \#$ \\
\hline $\mathrm{LAA}\left(\mathrm{cm}^{2}\right) \pm \mathrm{SD}$ & $25.6( \pm 5.6)$ & $26.9( \pm 8.1)$ & $31.8( \pm 7.8)$ & $P<0.001 \# \#$ \\
\hline LAA enlargement (\%) & 33.7 & 48.8 & 97.3 & $<0.001 ¥$ \\
\hline LV enlargement (\%) & 12.2 & 16.4 & 14.1 & NS \\
\hline HCM (\%) & 33.7 & 26.9 & 18.6 & $0.001 ¥$ \\
\hline$E F<35 \%(\%)$ & 2.3 & 11.9 & 6.2 & NS \\
\hline
\end{tabular}

Legend: ¥P < 0.05 LR vs HR; \#P < 0.05 MR vs HR; *P < 0.05 LR vs MR; IVSd - Interventricular septum thickness at end-diastole; LVED - Left ventricular end-diastolic diameter; LV mass - Left ventricle mass; RWT Relative wall thickness; LAd - Antero-posterior diameter of left atrium; LAA - Left atrial area HCM - Hypertrophic cardiomyopathy; EF - Ejection fraction

- due to a non-diagnostic acoustic window for echocardiography (two people).

Finally, the analysis included results of examinations of 663 patients with AIS, including $47.8 \%$ (317) of women and $52.2 \%$ (346) of men. The mean age of patients was 71 years (age range 20-97 years). A summary of the echocardiographic parameters is set out in Table 2.

$35.3 \%$ (234 patients) of examinations were carried out at the bedside, $26.2 \%$ (174) under difficult conditions, and $2.87 \%$ (19) in very difficult conditions. The most common abnormalities found in echocardiography were left ventricular hypertrophy and left atrial enlargement.

In the studied group, $65.2 \%$ of the patients (432 patients) had concentric left ventricular hypertrophy. In $20.1 \%$ of the patients (133), the concentric hypertrophy reached values that met the criteria for diagnosing hypertrophic cardiomyopathy based on the IVSd measurement in accordance with the recommendations of the European Society of Cardiology [17]. In $10.7 \%$ of the patients (71), eccentric hypertrophy of the left ventricle was found in the examination.
The incidence of enlargement of the left atrium depends on the used assessment method. The antero-posterior diameter (LAd) exceeded norms in $70.3 \%$ of the patients. Using the planimetric method (LAA - left atrial area), left atrial enlargement was found in $85.2 \%$ of the patients. Among this group of patients, 117 people (25.1\%) were diagnosed with atrial fibrillation.

\section{HR group}

The patients with a high risk of a cardiogenic stroke source constituted $26.7 \%$ of all the subjects (177 people).

The mean age in the high-risk group was 77.9 years. $57.1 \%$ of the patients (101 people) in this group were women. The main factor qualifying for the HR group was the occurrence of atrial fibrillation $(96 \%, 170$ people). In $64.4 \%$ of the patients (114 people) from the HR group, atrial fibrillation occurred during echocardiography. $31.6 \%$ of the patients (56 people) with a sinus rhythm during echocardiography were qualified for the HR group due to paroxysmal atrial fibrillation diagnosed earlier or during hospitalisation. 
In $11.9 \%$ of the patients ( 21 people) qualified for the HR group, non-AF factors of a high risk of a cardiogenic stroke source were detected, and in this group seven patients (four with an intracardiac thrombus, two with a mechanical valve, and one with a rheumatic defect) suffered from the co-occurrence of atrial fibrillation.

Finally, in 14 people (2.1\% of the entire group), factors of a high risk of a cardiogenic stroke source other than atrial fibrillation were found: thrombus in left heart cavities (six people), presence of left-sided mechanical valve (five people), and one person with infective endocarditis (IE), left ventricle non-compaction, and a rheumatic heart defect.

\section{MR group}

The patients with a moderate risk of a cardiogenic stroke source constituted $10.1 \%$ of all the subjects qualified for the examination (67 people). $50.7 \%$ (34 people) of the MR group were women. The mean age in the moderate risk group was 78.6 years.

The most common causes qualifying for the MR group included: occurrence of severe degenerative changes on left-sided valves ( 52 people, $77.6 \%$ ), including 34.3\% (23 people) with mitral annulus calcification (MAC). Other causes were less frequent: dilated cardiomyopathy (seven people), left ventricular post-infarction aneurysm and a biological valve (two people), spontaneous contrast in heart cavities (one person).

\section{LR group}

The patients with a low risk of a cardiogenic stroke (LR group) constituted $25.9 \%$ of all the subjects (172 people), of which women accounted for $37.8 \%$ ( 65 people). The mean age in this group was 70.5 years. One of the qualification criteria for this group was diagnosing persistent foramen ovale (PFO), which was detected in three people ( $0.45 \%$ of all the patients).

\section{Other results}

In $37.3 \%$ of the subjects ( 247 people) no potential causes of the cardiogenic stroke source were found. The mean age in the studied group was 65.3 years, and women accounted for $47 \%$ (116 people).

A group of 10 people (1.5\% of those who were included in the study) was identified among the patients, whose TTE image indicated the presence of a thrombus in the left heart cavities. The mean age in this group was 69.6 years, and nine out of 10 were men. The mean ejection fraction of patients with a thrombus in the heart cavity was $41 \%$. Based on the echocardiographic image, previous myocardial injury was found in eight patients from this group. Moreover, the group was characterised by the presence of many comorbidities such as arterial hypertension (six people), cardiomyopathy (two people), ventricular arrhythmias (two people), renal failure (two), and type 2 diabetes (three people).

\section{Discussion}

The research that we have conducted is one of the largest available in the literature on the subject, and the first one describing the Polish population and analysing the role of an echocardiographic assessment in patients hospitalised due to acute ischaemic stroke. The echocardiographic examination that is often recommended and performed in patients with AIS seems to have little effect on further proceedings in this group of patients.

During the study, only in $1.21 \%$ of all the patients did the TTE result influence a change in treatment. This percentage can be compared to the result obtained in similar research carried out at the Duke University Medical Centre, where the TTE result, subsequently confirmed by TEE, was associated with a change in treatment in $1.52 \%$ of patients (four people) [18]. The change in treatment mainly concerns patients with a thrombus in the heart cavity or myocardial non-compaction, without other indications for an anticoagulation therapy and patients with valvular heart diseases that require surgical treatment, including active IE.

Undoubtedly, the most frequent cause of formation of thromboembolic material in the heart cavities, which may become a cardiogenic stroke source, is atrial fibrillation [11]. AIS is the basic complication of AF that worsens the prognosis and is the cause of increased mortality in this group. Electrocardiographic examinations (resting, Holter) are used to diagnose AF. Due to the fact that all patients diagnosed with atrial fibrillation and AIS $(25.6 \%$ of the patients in the research) have indications for chronic anticoagulant therapy, in their case the result of the echocardiographic examination will rarely be associated with a change in secondary prevention of AIS [9]. A similar conclusion can be drawn from research that used transoesophageal echocardiography which was conducted at university hospitals in Berlin on patients after an ischaemic stroke, both with documented AF and without such a diagnosis. Only $3.8 \%$ of the patients with AF were diagnosed with embolic material during TEE, the origin of which could not be explained by co-occurring atrial fibrillation. However, this did not change the treatment in any of these patients, and the echocardiographic findings had no effect on the prescription of anticoagulants or a change in the anticoagulant therapy used at that time [19].

Considering the above results, performing TTE in patients with an ischaemic stroke and a diagnosed AF should be limited to people with additional disturbing symptoms such as a heart murmur or a fever suggestive of infective endocarditis.

Patients with AIS without any previously diagnosed atrial fibrillation or other indications for an anticoagulant therapy are a separate group. Although TTE is not a tool that allows a diagnosis of atrial fibrillation, it helps to distinguish those patients who are at risk of this arrhythmia. Left atrium enlargement, mitral valve defects, increased left ventricular hypertrophy, or the presence of pulmonary hypertension 
found during echocardiography are all recognised risk factors for paroxysmal AF, which can remain subclinical for a long time. The first manifestation of arrhythmia is sometimes an ischaemic stroke [20-27]. Assessing the size of the left atrium seems particularly useful. In our research, enlargement of the left atrium assessed by the surface area (LAA) occurred in 97.3\% of the patients in the HR group, whereas enlargement of the left atrium assessed with the use of LAd occurred in $83.5 \%$ of the patients in the HR group, in which people with AF predominated. Therefore, planimetric assessment of left atrium size should be preferred. Left atrial volume calculation, which is preferred in the guidelines, requires BSA calculation, which can be challenging in this population [28]. Patients with a significant left atrial enlargement could potentially benefit from prolonged electrocardiographic monitoring that would optimally last longer than seven days which, although recommended in guidelines, is still not available in Polish conditions [11].

Patients who have had a myocardial infarction and do not use an anticoagulation treatment are a special group that could benefit from echocardiographic assessment. In our research, of a group of 10 patients with thrombi visible in the TTE, eight had traits of post-infarction myocardial injury in the echocardiographic examination.

Another group that can also benefit from an echocardiographic examination is young patients with a cryptogenic stroke. In these patients, the detection of a patent persistent foramen ovale (PFO) with a documented right-left leak may be an indication for percutaneous closure to avoid recurrent AIS episodes $[6-8,29]$. The transthoracic examination allows a certain diagnosis of PFO only in a minority of cases. Therefore, in patients with suspected persistent foramen ovale, with a normal heart image in TTE, it is indicated to perform transoesophageal echocardiography (TEE) [11]. In the analysed group, only $0.45 \%$ of all the subjects during the TTE had persistent foramen ovale. Comparing this to the population occurrence of PFO, which amounts to $20-28 \%$, [30,31] shows a low diagnostic sensitivity of TTE in detecting PFO, especially in technically difficult bedside tests. Therefore, young people with a real cryptogenic strokewho are potentially eligible for PFO closure treatment should be qualified for a transoesophageal examination, which is usually made after an earlier transthoracic examination.

If a cardiogenic stroke source is suspected, it has been recommended that treatment start as early as possible to allow the diagnosis of a possible thrombus and to implement appropriate treatment as soon as possible [5]. The implementation of recommendations within 24 hours appears challenging. The limited availability of echocardiographic imaging, and the increasing use of echocardiographic laboratories, indicate a need for a careful selection of patients for this examination.

Communication between the neurologist ordering the treatment and the doctor performing the echocardiography is very important. The echocardiography order should contain basic information on the history of the cardiovascular system diseases, in particular: diagnosis of atrial fibrillation, previous myocardial infarction, and other factors that may suggest rare causes of cardiogenic attacks (e.g. fever in IE). Information on the cryptogenic nature of AIS, in particular in young people, may also be important. It is also useful to provide a patient's weight and height, if known, which allows some echocardiographic parameters to be referred to the patient's body surface.

The limitations to our publication include: difficult conditions of echocardiographic assessment resulting from the specifics of a studied group; lack of possibility to measure patients' height and weight due to their severe clinical conditions, and thus the lack of the possibility to scale the size of the heart cavities in relation to the patients' biometric size, including the assessment of the volume index recommended in the guidelines of the left atrial size parameter. Due to the lack of complete data, our publication did not include the nature of ischaemic changes in imaging (CT MRI) and clinical scales of stroke severity.

\section{Conclusions}

1. Transthoracic echocardiography performed routinely in all AIS patients affects the treatment in a very low percentage of cases.

2. The group that could benefit the most from TTE examination includes people without established indications for chronic anticoagulant therapy, in particular patients after myocardial infarction and people with additional clinical symptoms.

3. In patients with AIS, the diagnostic sensitivity of TTE in the detection of PFO is low. Young people with a cryptogenic ischaemic stroke should undergo a transoesophageal assessment.

This publication was prepared without any external sources of funding. The authors report no potential conflict of interest, which might include: grants funding the project; speaker honoraria from a company; consulting fees and stock options; other forms of gratification.

\section{References}

1. GBD 2013 Mortality and Causes of Death Collaborators. Global, regional, and national age-sex specific all-cause and cause-specific mortality for 240 causes of death, 1990-2013: a systematic analysis for the Global Burden of Disease Study 2013. Lancet. 2015; 385(9963): 117-171, doi: 10.1016/S0140-6736(14)61682-2, indexed in Pubmed: 25530442.

2. Celeste F, Muratori M, Mapelli M, et al. The Evolving Role and Use of Echocardiography in the Evaluation of Cardiac Source of Embolism. J Cardiovasc Echogr. 2017; 27(2): 33-44, doi: 10.4103/jcecho.jcecho_1_17, indexed in Pubmed: 28465991.

3. Staszewski J, Pruszczyk P. Diagnostyka udaru kardiogennego. Neurologia po dyplomie. 2015; 05: 34-43. 
4. Nakanishi K, Homma S. Role of echocardiography in patients with stroke. J Cardiol. 2016; 68(2): 91-99, doi: 10.1016/j.jjcc.2016.05.001, indexed in Pubmed: 27256218.

5. Olsen TS, Langhorne P, Diener HC, et al. European Stroke Initiative Executive Committee, EUSI Writing Committee. European Stroke Initiative Recommendations for Stroke Management-update 2003. Cerebrovasc Dis. 2003; 16(4): 311-337, doi: 10.1159/000072554, indexed in Pubmed: 14584488.

6. Kirchhof P, Benussi S, Kotecha D, et al. ESC Scientific Document Group. 2016 ESC Guidelines for the management of atrial fibrillation developed in collaboration with EACTS. Eur Heart J. 2016; 37(38): 2893-2962, doi: 10.1093/eurheartj/ehw210, indexed in Pubmed: 27567408.

7. Saxena R, Lewis S, Berge E, et al. Risk of early death and recurrent stroke and effect of heparin in 3169 patients with acute ischemic stroke and atrial fibrillation in the International Stroke Trial. Stroke. 2001; 32(10): 2333-2337, indexed in Pubmed: 11588322.

8. Kou S, Caballero L, Dulgheru R, et al. Echocardiographic reference ranges for normal cardiac chamber size: results from the NORRE study. Eur Heart J Cardiovasc Imaging. 2014; 15(6): 680-690, doi: 10.1093/ehjci/jet284, indexed in Pubmed: 24451180.

9. Camm AJ, Kirchhof P, Lip GYH, et al. U. Schotten U, Wytyczne dotyczace postępowania u chorych z migotaniem przedsionków. Kardiologia Polska. 2010; 68(supl. VII).

10. Weintraub RG, Semsarian C, Macdonald P. Dilated cardiomyopathy. Lancet. 2017; 390(10092): 400-414, doi: 10.1016/S01406736(16)31713-5, indexed in Pubmed: 28190577.

11. Saric M, Armour AC, Arnaout MS, et al. Guidelines for the Use of Echocardiography in the Evaluation of a Cardiac Source of Embolism. J Am Soc Echocardiogr. 2016; 29(1): 1-42, doi: 10.1016/j. echo.2015.09.011, indexed in Pubmed: 26765302.

12. Ay H, Benner T, Arsava EM, et al. A computerized algorithm for etiologic classification of ischemic stroke: the Causative Classification of Stroke System. Stroke. 2007; 38(11): 2979-2984, doi: 10.1161/ STROKEAHA.107.490896, indexed in Pubmed: 17901381.

13. Pepi M, Evangelista A, Nihoyannopoulos P, et al. European Association of Echocardiography. Recommendations for echocardiography use in the diagnosis and management of cardiac sources of embolism: European Association of Echocardiography (EAE) (a registered branch of the ESC). Eur J Echocardiogr. 2010; 11(6): 461-476, doi: 10.1093/ ejechocard/jeq045, indexed in Pubmed: 20702884.

14. Sacco R, Adams R, Albers G, et al. Guidelines for Prevention of Stroke in Patients With Ischemic Stroke or Transient Ischemic Attack. Stroke. 2006; 37(2): 577-617, doi: 10.1161/01.str.0000199147.30016.74.

15. Asinger RW, Dyken ML, Fisher M, et al. Cardiogenic brain embolism: The second report of the cerebral embolism task force. Archives of Neurology. 1989; 46(7): 727-743.

16. Dyken ML. Fisher M. Harrison MJG. et al. Cardiogenic brain embolism. Archives of Neurology. 1986; 43(1): 71-84.

17. Elliott $\mathrm{P}$, Anastasakis A, Borger M, et al. Wytyczne ESC dotyczące rozpoznawania i postępowania w kardiomiopatii przerostowej w 2014 roku. Kardiologia Polska. 2014; 72(11): 1054-1126, doi: 10.5603/ kp.2014.0212.

18. Zhang L, Harrison JK, Goldstein LB. Echocardiography for the detection of cardiac sources of embolism in patients with stroke or transient ischemic attack. J Stroke Cerebrovasc Dis. 2012; 21(7):
577-582, doi: 10.1016/j.jstrokecerebrovasdis.2011.01.005, indexed in Pubmed: 21367623.

19. Herm J, Konieczny M, Jungehulsing GJ, et al. Should transesophageal echocardiography be performed in acute stroke patients with atrial fibrillation? J Clin Neurosci. 2013; 20(4): 554-559, doi: 10.1016/j. jocn.2012.03.049, indexed in Pubmed: 23384509.

20. Casaclang-Verzosa G, Gersh BJ, Tsang TSM. Structural and functional remodeling of the left atrium: clinical and therapeutic implications for atrial fibrillation. J Am Coll Cardiol. 2008; 51(1): 1-11, doi: 10.1016/j. jacc.2007.09.026, indexed in Pubmed: 18174029.

21. Flaker GC, Fletcher KA, Rothbart RM, et al. Clinical and echocardiographic features of intermittent atrial fibrillation that predict recurrent atrial fibrillation. Stroke Prevention in Atrial Fibrillation (SPAF) Investigators. Am J Cardiol. 1995; 76(5): 355-358, indexed in Pubmed: 7639159.

22. Luo Y, Zhu Y, Guan X, et al. Assessment of mitral annulus and mitral leaflet in nonvalvular atrial fibrillation patients with various degrees of mitral regurgitation: Real time 3D transesophageal echocardiography. Echocardiography. 2018; 35(4): 481-486, doi: 10.1111/echo.13810, indexed in Pubmed: 29349803.

23. Olsson KM, Nickel NP, Tongers J, et al. Atrial flutter and fibrillation in patients with pulmonary hypertension. Int J Cardiol. 2013; 167(5): 2300-2305, doi: 10.1016/j.jijcard.2012.06.024, indexed in Pubmed: 22727973.

24. Papadopoulos $\mathrm{CH}$, Oikonomidis D, Lazaris E, et al. Echocardiography and cardiac arrhythmias. Hellenic J Cardiol. 2018; 59(3): 140-149, doi: 10.1016/j.hjc.2017.11.017, indexed in Pubmed: 29203161.

25. Park KM, Im SII, Kim EK, et al. Atrial Fibrillation in Hypertrophic Cardiomyopathy: Is the Extent of Septal Hypertrophy Important? PLoS One. 2016; 11(6): e0156410, doi: 10.1371/journal.pone.0156410, indexed in Pubmed: 27258035.

26. Ruiz-Cano MJ, Gonzalez-Mansilla A, Escribano P, et al. Clinical implications of supraventricular arrhythmias in patients with severe pulmonary arterial hypertension. Int J Cardiol. 2011; 146(1): 105-106, doi: 10.1016/j.jijcard.2010.09.065, indexed in Pubmed: 21056484.

27. Tongers J, Schwerdtfeger B, Klein G, et al. Incidence and clinical relevance of supraventricular tachyarrhythmias in pulmonary hypertension. Am Heart J. 2007; 153(1): 127-132, doi: 10.1016/j. ahj.2006.09.008, indexed in Pubmed: 17174650.

28. Lang RM, Badano LP, Mor-Avi V, et al. Recommendations for cardiac chamber quantification by echocardiography in adults: an update from the American Society of Echocardiography and the European Association of Cardiovascular Imaging. J Am Soc Echocardiogr. 2015; 28(1): 1-39.e14, doi: 10.1016/j.echo.2014.10.003, indexed in Pubmed: 25559473.

29. Hutyra M, Pavlů L, Šaňák D, et al. The role of echocardiography in patients after ischemic stroke. Cor et Vasa. 2016; 58(2): e261-e272, doi: 10.1016/j.crvasa.2016.02.003.

30. Hagen PT, Scholz DG, Edwards WD. Incidence and size of patent foramen ovale during the first 10 decades of life: an autopsy study of 965 normal hearts. Mayo Clin Proc. 1984; 59(1): 17-20, indexed in Pubmed: 6694427.

31. Johansson MC, Eriksson P, Dellborg M. The significance of patent foramen ovale: a current review of associated conditions and treatment. Int J Cardiol. 2009; 134(1): 17-24, doi: 10.1016/j.ijcard.2009.01.049, indexed in Pubmed: 19232760. 